PHYSICAL REVIEW D 84, 027901 (2011)

\title{
Space-time transformations of the Born-Infeld gauge field of a D-brane
}

\author{
Roberto Casalbuoni* \\ Department of Physics, University of Florence and INFN, Via G. Sansone 1, 50019 Sesto Fiorentino (FI), Italy \\ Joaquim Gomis ${ }^{\dagger}$ \\ Departament d'Estructura i Constituents de la Matèria and Departament de Física, Universitat de Barcelona, \\ Diagonal 647, 08028 Barcelona, Spain and C.E.R. for Astrophysics, Particle Physics, and Cosmology, Barcelona, Spain \\ and Department of Theoretical Physics, Australian National University, Canberra, ACT 0200, Australia \\ Kiyoshi Kamimura \\ Department of Physics, Toho University, Funabashi, Chiba 274-8510, Japan \\ (Received 4 May 2011; published 1 July 2011)

\begin{abstract}
Recently, Gliozzi has shown that, under certain conditions, it is possible to derive the Dirac-Born-Infeld action for an Abelian gauge field of a D-brane. Also, the action turns out to be invariant with respect to a nonlinear realization of the full Poincare group. A crucial role is played by the transformation properties of the gauge field under the nonlinear realization. The aim of this paper is to point out that these transformation properties are derived directly from the gauge fixing of the diffeomorphisms of the brane and the necessary compensating reparametrization when one performs a Lorentz transformation.
\end{abstract}

PACS numbers: $11.25 . \mathrm{Mj}$

In a very recent paper [1], Gliozzi has shown that it is possible to derive the Dirac-Born-Infeld action for a gauge field lying on a $d$-dimensional D-brane, by studying the nonlinear representation of the Poincaré group in a $D$-dimensional Minkowski space in terms of the $D-d$ transverse coordinates of the brane. A crucial point of the derivation lies in the transformation properties of the brane gauge field $A_{a}(a=0,1, \cdots, d-1)$. According to the author of Ref. [1], "Actually it appears to be no specific guiding principle (for deriving the transformations), nevertheless a trial and error method produced a surprisingly simple solution." Then, the author shows that these properties generate a nonlinear realization of the Lorentz group on the gauge field. The aim of this Brief Report is to observe that the transformation properties of the gauge field follow simply from the requirement of a gauge compensating transformation, necessary when one fixes the gauge identifying the longitudinal coordinates with the brane parameters. Notice that the gauge compensating transformation is also required in order to get the correct transformation properties for the transverse coordinates (Eq. (1) of [1]).

We begin considering, as in [1], a Poincaré invariant theory in a $D$-dimensional Minkowski space-time. Furthermore, we assume the presence of a stable $d$-dimensional extended object, a D-brane. We define coordinates $X^{\mu}(\mu=0,1, \ldots, D-1)$ in Minkowski space. $X^{\mu}\left(\sigma^{\alpha}\right)$ is a target space vector, depending on the brane coordinates $\sigma^{\alpha}(\alpha=0,1, \ldots, d-1)$. The covariant

\footnotetext{
*casalbuoni@fi.infn.it

gomis@ecm.ub.es

*kamimura@ph.sci.toho-u.ac.jp
}

theory must be defined in such a way as to be invariant under diffeomorphisms. Furthermore, the coordinates transform under global Lorentz transformations as

$$
\begin{gathered}
\delta_{L} X^{\mu}\left(\sigma^{\alpha}\right)=\omega^{\mu}{ }_{\nu} X^{\nu}, \quad \mu, \nu=0,1 \ldots, D-1, \\
\alpha=0,1, \ldots, d-1 .
\end{gathered}
$$

We assume also the presence on the brane of a Born-Infeld $d$-dimensional Abelian gauge field, $A_{\alpha}\left(\sigma^{\beta}\right)$, transforming as a scalar under the previous transformation

$$
\delta_{L} A_{\alpha}\left(\sigma^{\beta}\right)=0 .
$$

On the other hand, under local diffeomorphisms, $X^{\mu}$ and $A_{\alpha}$ should transform as a scalar and a vector, respectively,

$$
\begin{aligned}
\delta_{D} X^{\mu}\left(\sigma^{\beta}\right) & =\epsilon^{\beta} \partial_{\beta} X^{\mu}, \\
\delta_{D} A_{\alpha}\left(\sigma^{\beta}\right) & =\epsilon^{\beta} \partial_{\beta} A_{\alpha}+\partial_{\alpha} \epsilon^{\beta} A_{\beta}, \\
\epsilon^{\alpha} & =\epsilon^{\alpha}\left(\sigma^{\beta}\right) .
\end{aligned}
$$

Now, we fix the gauge with respect to local diffeomorphisms by letting

$$
X^{a}\left(\sigma^{\alpha}\right)=\sigma^{a} \equiv x^{a} .
$$

Notice that the index $\alpha$ transforms under diffeomorphisms, whereas the index $a$ transforms under the Lorentz group. With this choice of gauge, we identify the two kinds of indices.

Then, in this gauge,

$$
X^{\mu}=\left(X^{a}\left(\sigma^{b}\right), X^{i}\left(\sigma^{b}\right)\right)=\left(x^{b}, X^{i}\left(x^{b}\right)\right), \quad i=d, \ldots, D-1 .
$$

The transverse coordinates $X^{i}\left(x^{a}\right)$ describe the long wavelength fluctuations of the brane-that is, the Goldstone 
modes associated to the breaking of the translational invariance along the transverse direction specified by the index $i$. Of course, also the Lorentz invariance $S O(1, D-1)$ is broken to $S O(1, d-1) \otimes S O(D-d)$, but this breaking does not produce any further Goldstone boson; see, for example, [2]. This has to do with the inverse Higgs effect; see [3] and the more recent paper [4]. The Lorentz group $S O(1, D-1)$ is realized nonlinearly in terms of the transverse coordinates $X^{i}\left(x^{a}\right)$. We will show now how this comes about. First of all, let us notice that, under transformations of $S O(1, d-1)$, the coordinates $X^{a}$ transform linearly,

$$
\delta_{L} X^{a}=\omega_{b}^{a} X^{b} .
$$

Then, let us consider Lorentz transformations along the planes $(a, i)$, corresponding to the broken directions. In order to stay in the gauge chosen in (4), we have to perform, at the same time, a compensating diffeomorphism transformation such that

$$
\begin{aligned}
0 & =\left.\delta X^{a}\right|_{X^{a}=x^{a}}=\left.\left(\delta_{L} X^{a}+\delta_{D} X^{a}\right)\right|_{X^{a}=x^{a}} \\
& =\left.\left(\omega^{a}{ }_{i} X^{i}+\epsilon^{b} \partial_{b} X^{a}\right)\right|_{X^{a}=x^{a}}=\omega^{a}{ }_{i} X^{i}\left(x^{b}\right)+\epsilon^{a}\left(x^{b}\right),
\end{aligned}
$$

where $\epsilon^{a}$ is the compensating diffeomorphism parameter,

$$
\epsilon^{a}\left(x^{b}\right)=-\omega^{a}{ }_{i} X^{i}\left(x^{b}\right),
$$

ensuring that the gauge is preserved. Now, the Lorentz transformation in the plane $(a, i)$ must be supplemented by the compensating diffeomorphism parametrized by $\epsilon^{a}$. For the transverse coordinates, we get

$$
\begin{aligned}
\delta X^{i}\left(x^{a}\right) & =\omega_{a}^{i} X^{a}+\epsilon^{b} \partial_{b} X^{i}=\omega^{i}{ }_{a} x^{a}-\omega^{b}{ }_{j} X^{j} \partial_{b} X^{i} \\
& =-\omega^{b}{ }_{j}\left(\eta^{j i} x_{b}+X^{j} \partial_{b} X^{i}\right) .
\end{aligned}
$$

These transformations have been obtained, for example, in [5-7] and discussed in [1]. The identification of the indices $a$ and $\alpha$ implies that the gauge field $A_{a}$ transforms linearly as a vector under $S O(1, d-1)$ and undergoes an induced Lorentz transformation in the plane $(a, i)$, due to the diffeomorphism necessary to keep the gauge fixed.

$$
\begin{aligned}
\delta A_{a}(x) & =\epsilon^{b} \partial_{b} A_{a}+\partial_{a} \epsilon^{b} A_{b} \\
& =-\omega^{b}{ }_{i} X^{i}(x) \partial_{b} A_{a}-\left(\partial_{a} \omega^{b}{ }_{i} X^{i}(x)\right) A_{b} \\
& =-\omega^{b}{ }_{i}\left(X^{i} \partial_{b} A_{a}+\left(\partial_{a} X^{i}\right) A_{b}\right) .
\end{aligned}
$$

This equation is the same as Eq. (2) of [1]. Therefore, we have shown that the transformation properties of $A_{a}$ have the same geometrical basis as Eq. (9), expressing the transformation of the transverse coordinates, that is the need of the gauge compensating term. From this point of view, the fact that the field $A_{a}$ supports a nonlinear representation of the Lorentz group does not come as a surprise.

The transformations (6) and (7) in [1] of the world tensors $g_{\alpha \beta}$ and $F_{\alpha \beta}$ are also understood in the same manner, since they transform under diffeomorphisms as

$$
\begin{aligned}
& \delta g_{\alpha \beta}=\epsilon^{\gamma} \partial_{\gamma} g_{\alpha \beta}+\left(\partial_{(\alpha} \epsilon^{\gamma}\right) g_{\gamma \beta)}, \\
& \delta F_{\alpha \beta}=\epsilon^{\gamma} \partial_{\gamma} F_{\alpha \beta}+\left(\partial_{[\alpha} \epsilon^{\gamma}\right) F_{\gamma \beta]}
\end{aligned}
$$

and they are invariant under transformations in the plane $(a, i)$. Here, the small round (square) parentheses stay for symmetrization (antisymmetrization). Then, the gauge fixed $(a, i)$ transformations are

$$
\begin{aligned}
\delta g_{a b} & =\left(-\omega^{c}{ }_{i} X^{i}\right) \partial_{c} g_{a b}-\omega^{c}{ }_{i}\left(\partial_{(a} X^{i}\right) g_{c b)} \\
& =-\omega^{c}{ }_{i}\left(X^{i} \partial_{c} g_{a b}+\left(\partial_{(a} X^{i}\right) g_{c b)}\right), \\
\delta F_{a b} & =\left(-\omega^{c}{ }_{i} X^{i}\right) \partial_{c} F_{a b}-\omega^{c}{ }_{i}\left(\partial_{[a} X^{i}\right) F_{c b]} \\
& =-\omega^{c}{ }_{i}\left(X^{i} \partial_{c} F_{a b}+\left(\partial_{[a} X^{i}\right) F_{c b]}\right) .
\end{aligned}
$$

We would like also to stress the fact that the transformation of $A_{a}$ could have been obtained thinking of it as it would be a "matter field" by following the standard techniques of the nonlinear representations.

This can be seen in a more clear way by considering the general transformation of $X^{\mu}$ consisting in a translation plus a generic Lorentz transformation plus a diffeomorphism:

$$
\delta X^{a}(x)=\omega^{a}{ }_{b} X^{b}+\omega^{a}{ }_{i} X^{i}+\rho^{a}+\epsilon^{\beta} \partial_{\beta} X^{a},
$$

where $\rho^{a}$ are the parameters of the translations. To obtain the compensating diffeomorphism, we also require the variation of $X^{a}$ to be zero in the gauge $X^{a}(\sigma)=x^{a}$ $(a=0, \ldots, d-1)$. It gives

$$
\epsilon^{a}(x)=-\left(\omega^{a}{ }_{i} X^{i}+\omega^{a}{ }_{b} x^{b}+\rho^{a}\right) .
$$

The corresponding general transformations for $X^{i}, A_{a}$ are given by

$$
\begin{gathered}
\delta X^{i}(x)=-\omega^{b}{ }_{j}\left(\eta^{j i} x_{b}+X^{j} \partial_{b} X^{i}\right)+\omega^{i}{ }_{j} X^{j}+\rho^{i} \\
-\left(\omega^{b}{ }_{c} x^{c}+\rho^{b}\right) \partial_{b} X^{i}, \\
\delta A_{a}(x)=-\omega^{b}{ }_{i}\left(X^{i} \partial_{b} A_{a}+\left(\partial_{a} X^{i}\right) A_{b}\right) \\
+\omega_{a}{ }^{b} A_{b}-\left(\omega^{b}{ }_{c} x^{c}+\rho^{b}\right) \partial_{b} A_{a} .
\end{gathered}
$$

Note that, as expected, the gauge field transforms under longitudinal transformations. The second-to-last term of $\delta A_{a}$ in (16) is the rotation of $A_{a}$, and the last term indicates the $I S O(d)$ transformation of $x^{b}$ in $A_{a}(x)$.

The infinitesimal transformation (16) can be written as

$$
\begin{aligned}
& A_{a}^{\prime}\left(x^{b}+\omega^{b}{ }_{i} X^{i}+\omega^{b}{ }_{c} x^{c}+\rho^{b}\right)-A_{a}(x) \\
& \quad=\left(\omega_{a}{ }^{b}-\omega^{b}{ }_{i}\left(\partial_{a} X^{i}\right)\right) A_{b} .
\end{aligned}
$$

This transformation shows that the Born-Infeld field is a covariant field of the nonlinear realization of the Poincaré $\quad I S O(1, D-1) /(I S O(1, d-1) \otimes S O(1, D-d))$, if we consider that $A_{a}$ transforms under the vector 
representation of the $S O(1, d-1)$ subgroup of the unbroken group. Therefore, $A_{a}$ should not be considered as a Goldstone of some new space-time symmetry but rather as a covariant field of the nonlinear realization $[8,9]$ of the Poincaré group in $D$ dimensions.
Joaquim Gomis acknowledges Peter Bouwknegt for the warm hospitality at the Australian National University. We acknowledge partial financial support from projects FP2010-20807-C02-01, 2009SGR502, and CPAN Consolider CSD 2007-00042.
[1] F. Gliozzi, arXiv:1103.5377.

[2] J. Gomis, K. Kamimura, and P.C. West, Classical Quantum Gravity 23, 7369 (2006).

[3] E. A. Ivanov and V. I. Ogievetsky, Teor. Mat. Fiz. 25, 164 (1975).

[4] I. Low and A. V. Manohar, Phys. Rev. Lett. 88, 101602 (2002).

[5] P. Goddard, J. Goldstone, C. Rebbi, and C. B. Thorn, Nucl. Phys. B56, 109 (1973).
[6] O. Aharony, Z. Komargodski, and A. Schwimmer (unpublished).

[7] O. Aharony and M. Field, J. High Energy Phys. 01 (2011) 065.

[8] S. R. Coleman, J. Wess, and B. Zumino, Phys. Rev. 177, 2239 (1969).

[9] C. G. Callan, Jr., S. R. Coleman, J. Wess, and B. Zumino, Phys. Rev. 177, 2247 (1969). 